\title{
The extraction and mass transfer process of soluble solids in Russian olive
}

\author{
Kang Jian*, Wu Tong, Zhu Hongyuan and Sun Leilei \\ College of Life Sciences and Technology, Xinjiang University, Urumqi 830046, China. \\ Accepted 6 August, 2013
}

\begin{abstract}
In this paper, through the research of mass transfer mechanism of the extraction process of soluble solids (SS) in Russian olive, the kinetic model of concentration change for the SS with the solvent multiple $B(\mathrm{~mL} / \mathrm{g})$, extraction temperature $\mathrm{T}(\mathrm{K})$ and extraction time $\mathrm{t}(\mathrm{h})$ during extraction process was established for the first time. Russian olive extract concentration values $\hat{Y}$ (\%) can be calculated in different extraction process, and the extraction process can also be predicted and controlled if the extraction temperature, extraction time and a multiple of solvent are provided. Overall, the absolute errors of the kinetic model are less than $\pm 1 \%$ and the $X^{2}$ values are less than $x_{0.995}^{2}$ values also.
\end{abstract}

Key words: Russian olive, soluble solid, extraction, mass transfer mechanism.

\section{INTRODUCTION}

Russian olive (Elaeagnus angustifolia L.) is a species of Elaeagnus which is drought-resistant, saline-alkali-tolerant and widely distributed in north China, Kazakhstan, Russia, Poland, the United States, Canadian, etc (Ahmadiani et al., 2000). Russian Olive has the same clinical therapeutic effect with Smecta in treating acute infantile watery diarrhea by reducing both severity and durations of diarrhea disease, and superior to Smecta in decreasing volumes of watery diarrhea and ORS intake (Duolikun et al., 2007). It has been shown that the extract of Russian olive play the role of anti-diarrhea and inhibit intestinal propulsive after studying with experimental mice diarrhea and treatment (Keshan and Jianyun, 2007; Hossein and Mohammad, 2003; Field Guide to Plants of China, 1972).

Previous research (Jian and Jianyun, 2008) showed that different extraction temperature, time length and solvent multiples have significant effects on the extract concentration with certain extraction solvent. However, the mathematical relationship among them needs to be established and well explained. The change in regulations of extract concentration needs to be expounded. The established kinetic model of the mass-transfer process of soluble solids (SS) in Russian olive needs to be validated exactly. These problems mentioned above were solved in this paper.

\section{MATERIALS AND METHODS}

The raw material of the experiment is complete mature dry Russianolives. They were purchased from Kashi in Xinjiang. After selection, they were enucleated and crushed up, and the samples were kept in clean and dry bottles.

For each experiment, the samples of Russian-olives were soaked in purified water. According to the multiple solvent, extraction temperature and extraction time, the SS concentration in the solution was detected with the handheld refractometer in the different extraction conditions. Each experiment was carried out in triplicate.

The modeling of the extraction dynamics in Russian Olive

The balance relationship of the extraction system whose mechanism has not been completely removed is complex. But a widely adopted simple model, hypothesizes, is described as follows: an infusibility porous solid contains the amount of solute which cannot be absorbed by the solid. For the amount of solvent, 
solute contents is considered under the saturated solubility. If the solid has sufficiently contacted with the solvent for a long time, solute could completely dissolve and liquid concentration in the lacuna of solid will be equal to the concentration of the surrounding liquid. At this time, the liquid composition will be stable. Theoretically, the extraction process of solid includes three stages: 1) solvent is permeated into the solute and solute begins to dissolve; 2) dissolved solute diffuses across the inside of the solid to the interface; 3) solute continues to diffuse from the interface across the liquid film to the main body of the external solvent.

Among the three stages, stage 1 and 3 which are not the crucial factors can be neglected while the stage 2 is vital for the extraction speed because the solute concentration changed during the extraction process and it decreased with the passing of time, so this process is actually a unstable diffusion according to the Fick's Law. In fact, the extraction operation depends on the control of internal diffusion.

Fick's first law relates the diffusive flux to the concentration field, by postulating that the flux goes from regions of high concentration to regions of low concentration, with a magnitude that is proportional to the concentration gradient (spatial derivative). In one (spatial) dimension, this is:

$$
J_{A}=-D_{A B} \frac{d c_{A}}{d z}
$$

Where $J_{A}$ is the diffusion flux in dimensions of ((amount of component_A) length ${ }^{-2}$ time $\left.^{-1}\right)$, example $\left(\mathrm{kmol} / \mathrm{m}^{2} \cdot \mathrm{s}\right) ; c_{A}$ is the concentration in dimensions of ((amount of component $A)$ length $\left.{ }^{-3}\right)$, example $\left(\mathrm{kmol} / \mathrm{m}^{3}\right) ; z$ is the position (length), example $(\mathrm{m}) ; D_{A B}$ is the diffusion coefficient or diffusivity in dimensions of (length ${ }^{2}$ time $\left.\mathrm{e}^{-1}\right)$, example, $\left(\mathrm{m}^{2} / \mathrm{s}\right)$; is $\frac{d c_{A}}{d z}$ is the driving force for the onedimensional diffusion of component t A, example $\left(\mathrm{kmol} / \mathrm{m}^{3} \cdot \mathrm{m}\right)$.

Generally, total concentration $c$ is not constant when main body flow takes place. Fick's law can be described as follows (Jian and Jianyun, 2008):

$$
J_{A}=-c D_{A B} \frac{d x_{A}}{d z}
$$

Where, $x_{A}$ is the mole fraction of component $A,(\%), x_{A}=C_{A} / C ; c_{A}$ is the the molarity of component $A,\left(\mathrm{kmol} / \mathrm{m}^{3}\right), c_{A}=m_{A} / M_{A} V ; c$ is the total molarity of compound $\left(\mathrm{kmol} / \mathrm{m}^{3}\right), c=\sum_{i=1}^{n} c_{i}$

In a multicomposition mixture, total molarity equals the sum of every component's molarity. $m_{A}$ is the quality of component $A(\mathrm{~kg})$; $M_{A}$ is the molecular weight of component $A ; V$ is the volume of the compound $\left(\mathrm{m}^{3}\right)$

To the diffusion of the SS in Russian olive in water, for the sake of convenience, considering the diffusion area, the Fick's first law used for describing the extraction process can be derived as follows (Yaping and Weilun, 1997):

$$
\frac{d m}{d t}=D S \frac{d \rho}{d z}
$$

Where, $\frac{d m}{d t}$ is the leaching rate of SS, example $(\mathrm{g} / \mathrm{h}) ; D$ is the diffusion coefficient $\left(\mathrm{m}^{2} / \mathrm{s}\right) ; S$ is diffusion area $\left(\mathrm{m}^{2}\right) ; \frac{d \rho}{d z}$ is the volume concentration gradient of solute at the interface $\left(\mathrm{g} / \mathrm{m}^{3} . \mathrm{m}\right)$.

The diffusion coefficient $D$ is related to the properties of the materiel and extraction temperature.

The relationship between $D$ and extraction temperature can be described by equation of Arrhniues (Jiufang et al., 1993) in a detailed extraction process:

$D=A e^{-E / R T}$

Where, $A$ is the the pre-exponential factor $\left(\mathrm{m}^{2} / \mathrm{s}\right) ; E$ is the diffusion activation energy $(\mathrm{J} / \mathrm{mol}) ; R$ is the gas constant $(\mathrm{J} / \mathrm{mol} \cdot \mathrm{K}) ; T$ is the absoluted temperature $(\mathrm{K}) ; E$ and $R$ are constant, pre-exponential factor $\mathrm{A}$ can be regarded as the diffusion coefficient at some basic temperature. Diffusion coefficient $D$ is not only related to temperature but also to the concentration of solute.if (Aikemu and Xinjiang, 2007):

$$
A=D_{0} \rho^{n}
$$

Where, $\rho$ is the volume concentration of solute in the main solvent $(\mathrm{g} / \mathrm{mL})$.

The effects of concentration on diffusion flux can be reflect by (5) with the change of index $n$. In a general way, diffusion coefficient decreases with the increase of the concentration of solute, therefore, $n<0$.

$D_{0}$ which is neither related to the temperature nor to the concentration, is only related to character of material, called natural diffusion coefficient.

The extraction is carried under the stable condition, and the particular concentration of diffusion surface is changing with time during the extraction process, as a result, concentration gradient is the function of not only the space but also the time.

Factually, as to the special diffusion surface, the larger the concentration gradient, the larger the diffusion force and the quicker the diffusion proceeds.

If the changing rate of time of the concentration gradient is indirect proportion to the temporal concentration gradient (Aikemu and Xinjiang, 2007):

$$
\frac{d}{d t}\left(\frac{d \rho}{d z}\right)=\alpha \frac{d \rho}{d z}
$$

Where, $\alpha$ is the rate constant and concentration gradient decreases with the hoist of concentration of solute and the process. $\alpha<0$.

if $t=0, \frac{d \rho}{d z}=u_{0}$, and $t=t, \frac{d \rho}{d z}=u$, the integral of (6) is:

$\int_{u_{0}}^{u} \frac{d u}{u}=\int_{0}^{t} \alpha d t$

The relationship between concentration gradient and time is:

$\frac{d \rho}{d z}=u_{0} e^{\alpha t}$

Put (4), (5), (7) into (3), then we get: 
$\frac{d m}{d t}=D_{0} u_{0} \rho^{n} S e^{-E / R T} e^{\alpha t}$

(8) is the amended Fick's first law.

SS exists in the flesh tissue under the skin of the Russian olive which has long olivary shape, and the thickness of the skin is extremely shorter than the length and diameter of the Russian olive itself. Therefore, the extraction process of SS can be simplified as single dimension diffusion under the condition of long flat, and formula (8) can be used to describe the extraction process. At this moment, the diffusion area $S$ of $S S$ in Russian olive is in direct proportion to absolutely dry biomass $G$

$S=k G$

Where $k$ is the surface area of Russian olive in each quality $\left(\mathrm{m}^{2} / \mathrm{g}\right)$

Total amounts $m$ of SS at T moment has something to do with volume concentration of solute and volume of solvent in the main solvent:

$m=\rho V$

where $\rho$ is the volume concentration of solute in main solvent $(\mathrm{g} / \mathrm{mL}) ; V$ is the volume of solvent $(\mathrm{mL})$

Put (9), (10) into (8):

$\frac{d \rho}{d t}=D_{0} u_{0} \rho^{n} k \frac{G}{V} e^{-E / R T} e^{\alpha t}$

We set solvent multiples as $B=\frac{V}{G}$, then:

$\frac{d \rho}{d t}=D_{0} u_{0} \rho^{n} k \frac{1}{B} e^{-E / R T} e^{\alpha t}$

If $t=0, \rho=0$, the integral of $(11)$ is:

$\int_{0}^{\rho} \frac{d \rho}{\rho^{n}}=D_{0} u_{0} k \frac{1}{B} e^{-E / R T} \int_{0}^{t} e^{\alpha t} d t$

$\rho^{1-n}=\frac{1-n}{\alpha B} D_{0} u_{0} k e^{-E / R T}\left(e^{\alpha t}-1\right)$

For $n<0, \alpha<0$, formula (12) can be changed as follows:

$\rho^{1-n}=\frac{n-1}{\alpha B} D_{0} u_{0} k e^{-E / R T}\left(1-e^{\alpha t}\right)$

The calculated value of sugar content $\hat{Y}(\%)$, which is also called Brix, can be used to reflect the concentration of SS in Russian olive. In fact, while using handheld sugar meter, refraction of material is determined.

And refraction is the quotient of the sine of angle of incidence and sine of angle of refraction. For the same material, the refraction is in direct proportion to its concentration.

According to the definition of the concentration of SS, $\hat{Y}$ (\%) is:
$\hat{Y}=\frac{m \times 100}{m+m_{2}}=\frac{m \times 100}{m+D_{2} V_{2}}$

Where, $m_{2}$ is the quality of the solvent (water) $(\mathrm{g}) ; D_{2}$ is the density of the solvent (water), $D_{2}=1(\mathrm{~g} / \mathrm{mL}) ; V_{2}$ is the volume of the solvent (water), $\left(\mathrm{mL}\right.$ ); because $\mathrm{m}<<\mathrm{m}_{2}, \mathrm{~m}_{2}=\mathrm{D}_{2} \mathrm{~V}_{2}=\mathrm{V}_{2}$ and $V_{2} \approx V$, (14) could be recomposed:

$\hat{Y}=\frac{m \times 100}{V}=100 \rho$

Put (15) into (13):

$\hat{Y}^{1-n}=\frac{100^{1-n}(n-1)}{\alpha} D_{0} u_{0} k \cdot \frac{1}{B} \cdot e^{-E / R T} \cdot\left(1-e^{\alpha t}\right)$

or:

$\ln \hat{Y}=\frac{1}{1-n} \ln \frac{100^{1-n}(n-1)}{\alpha} D_{0} u_{0} k-\frac{1}{1-n} \ln B-\frac{E}{R(1-n)} \frac{1}{T}+\frac{1}{1-n} \ln \left(1-e^{\alpha t}\right)$

In formula (16), $n, D_{0}, k, \alpha, E, R$ are constant. At the time $(t=0)$ when extraction begins, the solute concentration is 0 , so there is no relationship between concentration gradient $u_{0}$ and added solvent and the concentration gradient is only related to primary content of solute in solids, characteristics of solutes and solvents. Therefore, in the process of extraction of SS in Russian olive, $u_{0}$ is also a constant.

If

$\beta_{0}=\frac{1}{1-n} \ln \frac{100^{1-n}(n-1)}{\alpha} D_{0} u_{0} k$

$\beta_{1}=-\frac{1}{1-n}$

$\beta_{2}=-\frac{E}{R(1-n)}$

Then formula (16) could be recomposed:

$\ln \hat{Y}=\beta_{0}+\beta_{1} \ln B+\beta_{2} \frac{1}{T}-\beta_{1} \ln \left(1-e^{\alpha t}\right)$

Formula (20) is the dynamic pending model which can reflect the relationship between extraction concentration $\hat{Y}$ and extraction multiples $B$, temperature $T$ and time $t$. From formula (20) we could get the formulas of extraction concentration and 3 factors respectively.

2) When only the extraction multiplied $B$ is taken into account, extraction temperature $T$ and time $t$ is kept stable, formula (20) could be recomposed:

$\ln \hat{Y}=\beta_{B}+\beta_{1} \ln B$

$\beta_{B}$ - the constant when extraction multiples $B$ is taken into account. 
Table 1. Verification of soluble solids concentration in different solvent multiples ( $T=353 \mathrm{~K}, t=1.5 \mathrm{~h})$.

\begin{tabular}{lccccccccc}
\hline $\boldsymbol{B}$ & $\mathbf{4}$ & $\mathbf{5}$ & $\mathbf{6}$ & $\mathbf{7}$ & $\mathbf{1 0}$ & $\mathbf{1 3}$ & $\mathbf{1 7}$ & $\mathbf{2 0}$ & $\mathbf{3 0}$ \\
\hline Model calculation $\left(\hat{Y}_{i}\right)$ & 15.5 & 12.7 & 10.9 & 9.5 & 7.0 & 5.5 & 4.4 & 3.8 & 2.7 \\
Test value $\left(Y_{\mathrm{i}}\right)$ & 15.0 & 12.5 & 10.5 & 9.0 & 7.0 & 5.0 & 4 & 3.5 & 2.5 \\
Simulated absolute error & 0.5 & 0.2 & 0.4 & 0.5 & 0 & 0.5 & 0.4 & 0.3 & 0.2 \\
$\left(\hat{Y}_{i}-Y_{\mathrm{i}}\right)^{2} / Y_{\mathrm{i}}$ & 0.016 & 0.003 & 0.015 & 0.028 & 0.000 & 0.050 & 0.040 & 0.026 & 0.016 \\
\hline
\end{tabular}

$X^{2}=\sum\left(\hat{Y}_{i}-Y_{\mathrm{i}}\right)^{2} / Y_{\mathrm{i}}=0.194<\mathrm{X}^{2} 0.995 .(6)=0.680, \mathrm{P}>0.995$

It is related to temperature T and time t. the extraction test of SS in Russian Olive was carried out when keep the temperature $T$ and time $t$ stable and the test value $Y_{i}$ of the extraction concentration at different extraction multiples was gotten. Then $\beta_{B}$ and $\beta_{1}$ can be calculated by the liner regression between $\ln \hat{Y}$ and $\ln B$.

2) When only the extraction temperature $T$ is taken into account, extraction multiples $B$ and time $t$ is kept stable, formula (20) could be recomposed:

$\ln \hat{Y}=\beta_{T}+\beta_{2} \frac{1}{T}$

$\beta_{T^{-}}$the constant when extraction temperature $T$ is taken into account. It is related to extraction multiplied by $B$ and time $t$.

the extraction test of SS in Russian Olive was carried out when keeping the extraction multiple $B$ and time $t$ stable and the test value $Y_{i}$ of the extraction concentration at different extraction temperature gotten. Then $\beta_{T}$ and $\beta_{2}$ can be calculated by the liner regression between $\ln \hat{Y}$ and $\frac{1}{T}$.
3) When only the extraction time $t$ is taken into account, extraction multiples $B$ and temperature $T$ is kept stable, formula (20) could be gotten:

$\ln \hat{Y}=\beta_{t}-\beta_{1} \ln \left(1-e^{\alpha t}\right)$

$\beta_{t}$ - the constant when extraction time $t$ is taken into account. It is related to extraction multiples $B$ and temperature $T$.

The extraction test of SS in Russian Olive is carried out when keeping the extraction multiples $B$ and temperature $T$ stable and getting the test value $Y_{i}$ of the extraction concentration at different extraction time.

The value of $\beta_{t}$ and $\alpha$ can be calculated by least squares and fitting iterative methods with $Y_{i}$. The calculation steps are omitted. Lastly, the calculated results are:

$\beta_{1}=-0.8701 ; \beta_{2}=-1322.2725 ; \alpha=-0.6655 ; \beta_{0}=8.0913$

Put the final $\beta_{0}, \beta_{1}, \beta_{2}, \alpha$ into (20), we get the model of extraction dynamics of SS in Russian Olive:

$$
\ln \hat{Y}=8.0913-0.8701 \ln B-1322.2725 \frac{1}{T}+0.8701 \ln \left(1-e^{-0.6655 t}\right)
$$

This model can accurately reflect the relationship between the extract concentration and solvent multiples, extraction temperature and extraction time. If the other parameter such as solvents, size of particles and resource of materials are ensured, SS in Russian olive under variety technology could be predicted. However, the veracity of the prediction needs valuing.

\section{RESULTS AND VERIFICATION}

A comparison of predicted and experimental SS concentration in different solvent multiples of whole Russian olive during extraction at temperature of $80^{\circ} \mathrm{C}$ and time of $1.5 \mathrm{~h}$ is shown in Table 1 and Figure 1.

Every point in the experiment curve of each figure is the mean of SS concentration that was tested three times and the standard deviation was less than 0.3. It shows that the calculation gotten from formula (21) for SS concentration fits very well with the experiment $(x 2=0.68$, $\mathrm{P}>0.995)$.

A comparison of predicted and experimental of SS concentration in different extraction temperature of whole Russian Olive during extraction at the solvent multiple $B$ of 6 and time of $1.5 \mathrm{~h}$ is shown in Table 2 and Figure 2.
Every point in the experiment curve of each figure is the mean of SS concentration that was tested three times and the standard deviation was less than 0.3. It shows that the calculation gotten from formula (22) for SS concentration fit very well with the experiment $(x 2=0.68$, $P>0.995)$.

A comparison of predicted and experimental of SS concentration in different extraction time of whole Russian Olive during extraction at the solvent multiple $B$ of 6 and temperature of $80^{\circ} \mathrm{C}$ is shown in Table 3 and Figure 3.

Every point in the experiment curve of each figure is the mean of SS concentration that was tested three times and the standard deviation was less than 0.3. It shows that the calculation gotten from formula (23) for SS concentration fit very well with the experiment $\left(X^{2}=2.60\right.$, $P>0.995)$.

By analyzing the dates from tables and figures, the well simulated results demonstrated that the model was suitable for the extraction and mass transfer process of SS in Russian Olive. Between the model calculations and the test values, the absolute errors of SS concentration are less than $\pm 1 \%$ and their $x^{2}$ values are less than 


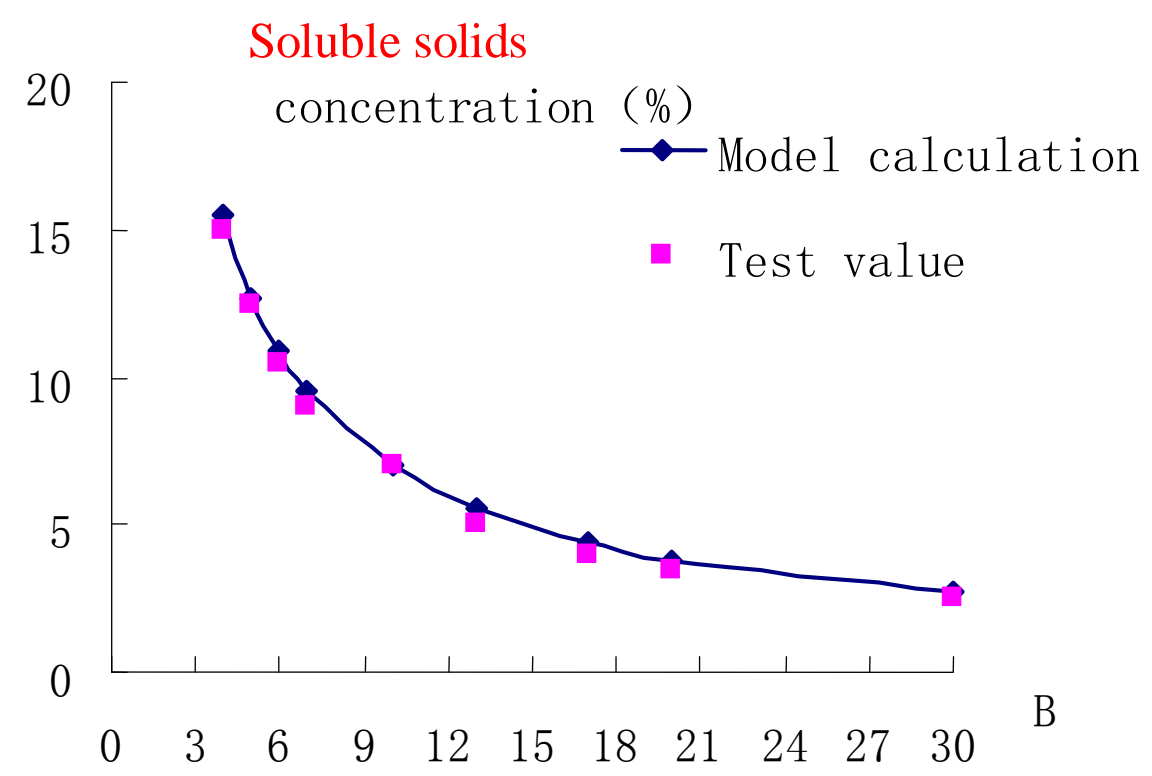

Figure 1. Verification of soluble solids concentration in different solvent multiples ( $T$ $=353 \mathrm{~K}, \mathrm{t}=1.5 \mathrm{~h})$.

Table 2. Verification of soluble solids concentration in different extraction temperature $(B=6, t=1.5$ h).

\begin{tabular}{lccccccc}
\hline $\boldsymbol{T}$ & 303K & $\mathbf{3 1 3 K}$ & $\mathbf{3 2 3 K}$ & $\mathbf{3 3 3 K}$ & $\mathbf{3 4 3 K}$ & $\mathbf{3 5 3 K}$ & $\mathbf{3 6 3 K}$ \\
\hline Model calculation $\left(\hat{Y}_{i}\right)$ & 5.9 & 6.7 & 7.7 & 8.7 & 9.8 & 10.9 & 12.1 \\
Test value $\left(Y_{\mathrm{i}}\right)$ & 6.2 & 7.2 & 8.2 & 9 & 10.2 & 11.5 & 13 \\
Simulated absolute error & -0.3 & -0.5 & -0.5 & -0.3 & -0.4 & -0.6 & -0.9 \\
$\left(\hat{Y}_{i}-Y_{\mathrm{i}}\right)^{2} / Y_{\mathrm{i}}$ & 0.015 & 0.037 & 0.032 & 0.010 & 0.016 & 0.033 & 0.067 \\
\hline
\end{tabular}

$\mathrm{X}^{2}=\sum\left(\hat{Y}_{i}-Y_{\mathrm{i}}\right)^{2} / Y_{\mathrm{i}}=0.210<\mathrm{X}^{2} 0.995 .(6)=0.680, \mathrm{P}>0.995$

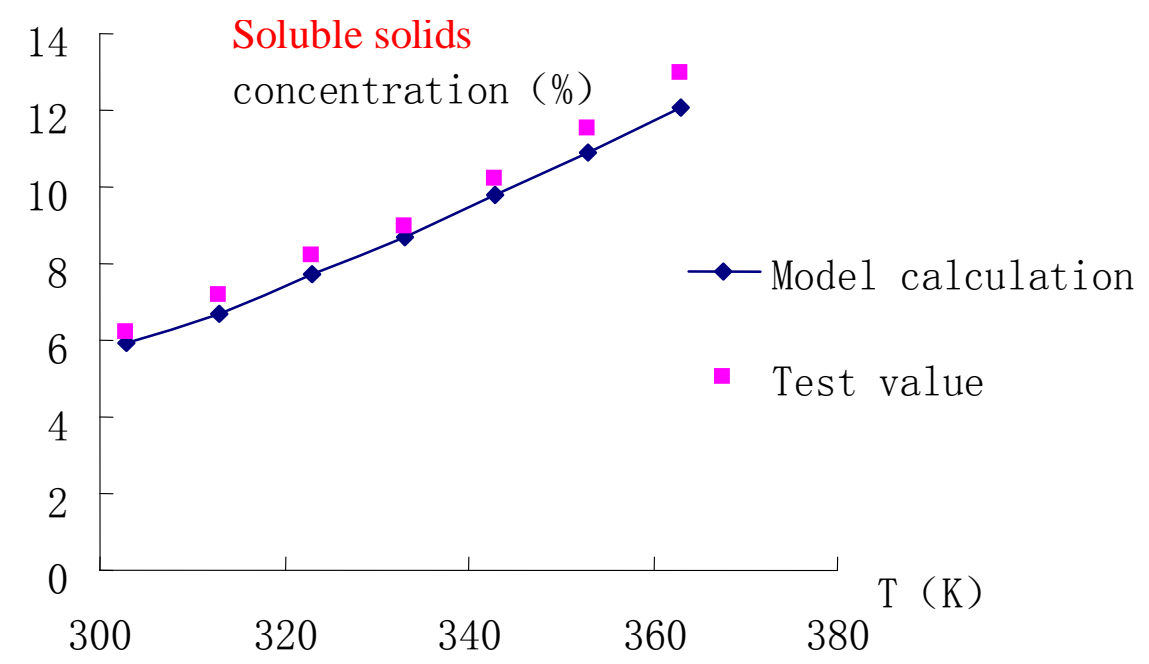

Figure 2. Verification of soluble solids concentration in different extraction temperature $(B=6, t=1.5 \mathrm{~h})$. 
Table 3. Verification of soluble solids concentration in different extraction time ( $B=6, T=353 \mathrm{~K})$.

\begin{tabular}{|c|c|c|c|c|c|c|c|c|c|c|c|c|c|}
\hline$t(\min )$ & 10 & 20 & 30 & 40 & 50 & 60 & 70 & 80 & 90 & 100 & 110 & 120 & 130 \\
\hline$t / \mathrm{h}$ & 0.1667 & 0.3333 & 0.5 & 0.6667 & 0.8333 & 1.0 & 1.1667 & 1.3333 & 1.5 & 1.6667 & 1.8333 & 2.0 & 2.1667 \\
\hline Model calculation $\left(\hat{Y}_{i}\right)$ & 2.3 & 4.0 & 5.4 & 6.6 & 7.7 & 8.7 & 9.5 & 10.2 & 10.9 & 11.5 & 12.0 & 12.4 & 12.8 \\
\hline Test value $\left(Y_{\mathrm{i}}\right)$ & 2.1 & 4.3 & 5.0 & 6.2 & 7.3 & 8.5 & 9.0 & 9.5 & 10.5 & 11.3 & 11.5 & 12.0 & 12.2 \\
\hline Simulated absolute error & 0.2 & -0.3 & 0.4 & 0.4 & 0.4 & 0.2 & 0.5 & 0.7 & 0.4 & 0.2 & 0.5 & 0.4 & 0.6 \\
\hline$\left(\hat{Y}_{i}-Y_{\mathrm{i}}\right)^{2} / Y_{\mathrm{i}}$ & 0.019 & 0.021 & 0.030 & 0.024 & 0.021 & 0.005 & 0.026 & 0.048 & 0.015 & 0.003 & 0.021 & 0.013 & 0.028 \\
\hline
\end{tabular}

$X^{2}=\sum\left(\hat{Y}_{i}-Y_{i}\right)^{2} / Y_{i}=0.274<X_{0.995 .(11)}^{2}=2.60, P>0.995$.

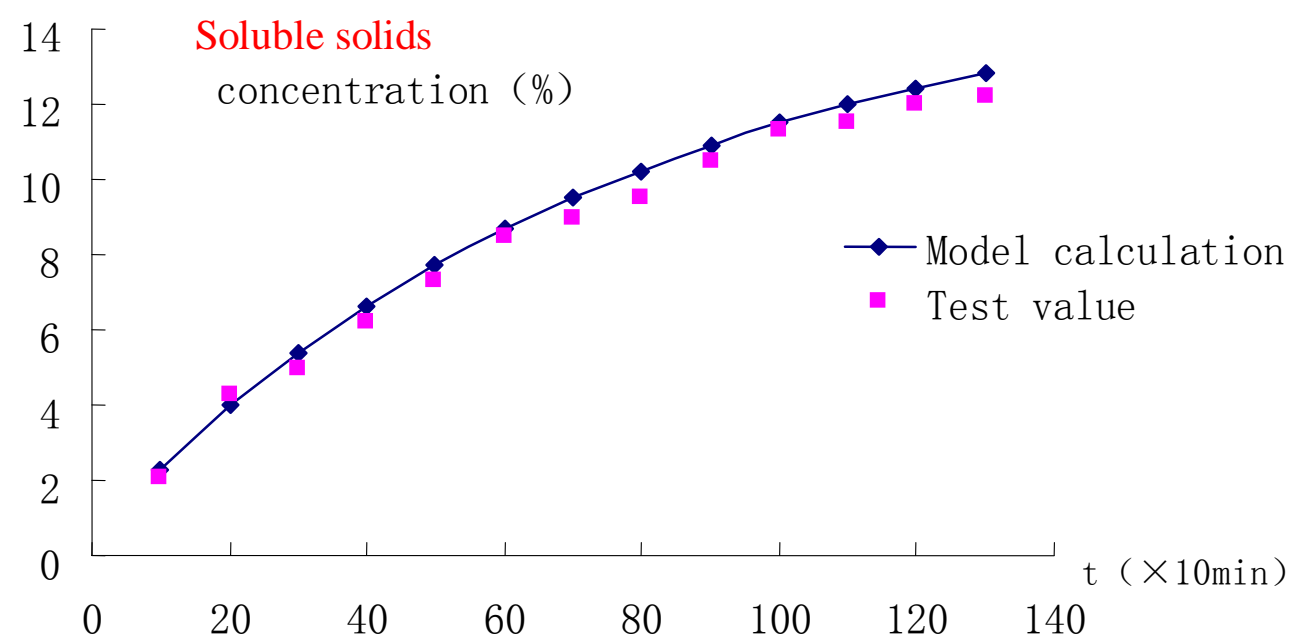

Figure 3. Verification of soluble solids concentration in different extraction time $(B=6, T=$ 353K).

$\mathrm{X}^{2}{ }_{0.995}$ values in different extraction temperature $T, \quad$ Conclusion

extraction time $t$, and solvent multiples $B$.

To sum up, the model can predict accurately to a large extent, and describe well the extraction dynamic process of SS concentration of Russian Olive, as well as reflect the correct relationship between SS concentration and extraction temperature, extraction time and solvent multiples.
1) According to the Fick's first law, we assume that the change rate of concentration gradient is in direct proportion to concentration gradient, and the relationship between diffusion coefficient and temperature satisfy the Arrhuiues equation. The model of extraction dynamic of SS in Russian olive has been derived theoretically. The characteristics of Russian olive and its SS are all reflected as constants in this model and such constants can be calculated through the experimental results. Therefore, the model can be applied to describe the other extraction kinetics process in similar dried fruits.

2) Based on the extraction experiments, according 
to mathematic formula between the SS concentration and extraction temperature, time, solvent multiples, we get the unknown constant in the model by adopting liner regression analytic method and least square method. The model calculation is a high precision with the test values at various extraction conditions as compared to the traditional orthogonal experiment method. Between the model calculations and the test values, the absolute error of SS concentration is less than $\pm 1 \%$ and their $x^{2}$ values are less than $X^{2}{ }_{0.995}$ values in different extraction temperature $\boldsymbol{T}$, extraction time $t$, and solvent multiples $B$.

3) The effect of extraction temperature, time, solvent multiples on SS concentration has been studied and the corresponding regulation has been gotten. Generally, the lower the multiples, the higher the temperature, the longer the time and the higher the SS concentration. However, there exist limitations if we keep reducing multiples and increasing time to some extent, the increase of SS concentration is limited. So there exist the most economic extraction time and solvent multiples, but the specific level is connected with extraction temperature.

4) When compared with Fick's law, this model is used simple and convenient. Under the same conditions (solvents, origin of materials, material maturity, etc), Russian olive extract concentration values $\hat{Y}(\%)$ can be calculated in different extraction process, and the production process of extraction can also be predicted and controlled if the extraction temperature $T(\mathrm{~K})$, extraction time $t(\mathrm{~h})$ and a multiple of solvent $B(\mathrm{~mL} / \mathrm{g})$ are provided. After all, the model can predict accurately to a large extent, and describe well the extraction dynamic process of SS concentration of Russian olive.

\section{REFERENCES}

Ahmadiani A, Hosseiny J, Semnanian S (2000). Antinociceptive and anti-inflammatory effects of Elaeagnus angustifolia fruit extract. $\mathrm{J}$. Ethnopharmacol. 72(1-2):287-292.

Aikemu M (2007). Xinjiang Elaeagnus angustifolia extract effect on experimental mice in diarrhea and small intestine propulsive function. Xinjiang Medical 37(3):1-3.

Duolikun M (2007). A clinical study on the treatment of Elaeagnus angustifolia in infants with acute watery diarrhea. J. Pediatr. Pharm. 13(1):18-20.

Field Guide to Plants of China (1972). Institute Botany, Chinese Sciences Academy (M).Beijing: Science Press.

Hossein H, Mohammad R (2003). Muscle relaxant activity of Elaeagnus angustifolia L. fruit seeds in mice. J. Ethnopharmacol. 84(2-3):275278.

Jian K, Jianyun C (2008). Study on the factors of extracting soluble solid from the Russian-olive in Xinjiang (J ). Food Res. Dev. 10(11):59-61.

Jian K, Jianyun C (2008). Comparison of the nutritional compositions in the Russian-olive from Xinjiang different area $(\mathrm{J})$. Food Res. Dev. 10(12):133-174.

Jiufang L, Zongcheng L, Tiezhu B (1993). Chemical Separation Process (M). Beijing: Tsinghua University Press. pp. 25-34.

Keshan G, Jianyun C (2007). Introduction to Food Engineering (M). Beijing: Chemical Industry Press. pp. 79-80.

Yaping L, Weilun L (1997). Emancipation Dynamic Model of noncorrosion-typy Drugs System (J). J. Pharm. 32(11):869-873. 